Chapter 3

\title{
Otorhinolaryngologic Manifestations in Leishmaniasis
}

\author{
Luiz Alberto Alves Mota and \\ Roberta Correia Ribeiro Ferreira de Miranda \\ Additional information is available at the end of the chapter \\ http://dx.doi.org/10.5772/57303
}

\section{Introduction}

Leishmaniasis is considered by the World Health Organization (WHO) as one of the five endemic infectious and parasitic diseases of greatest importance and as a world-wide public health problem [1]. It is an infectious disease that evolves chronically and is caused by a protozoon of the genus Leishmania, which may appear as a clinical form which is visceral, cutaneous, mucocutaneous, mucousal and rarely diffuse [2]. The term Leishmaniasis refers to the infection of vertebrate hosts Leishmania, which, like the other trypanosomatids of the order Kinetoplastida, characteristically present an extranuclear DNA in its cytoplasm in a mitochondrial organelle, the kinetoplast. This genus is characterized by having two ways of evolving during its biological cycle in host organisms: amastigote, which is an obligatory intracellular parasite in vertebrates, and promastigote, which develops in the digestive tube of invertebrate vectors or in axenic culture media [3].

In 1903, the agent of the disease was first described and separately by Leishman and Donovan. It is a protozoon identified in splenic tissue from two patients resident in India affected by a fatal disease [4]. It is primarily a zoonotic infection of wild animals, and more rarely pets, including marsupials, carnivores and even primates, with humans being accidental hosts.

All species of Leishmania are transmitted by the bite of female mosquitoes called phlebotominae of the genera Lutzomyia and Phlebotomus, this transmission being made by inoculation of promastigotes into the skin of the vertebrate host [5]. In larynegeal Leishmaniasis, contamination generally occurs starting with high lesions of the nasal cavities and oropharynx by contiguity. It is rare for parasites to be found inside the lesions [6].

The incubation period ranges from 2 weeks to several months [13]. Mucosal involvement is dependent on the combination of the virulence of the parasite and the immune cell response of the host. Within the population of infected individuals, $1-10 \%$ experience mucosal involve- 
ment [15]. Risk factors for the development of mucosal Leishmaniasis are: the presence of lesions above the pelvis, large skin ulcers and inadequate treatment of cutaneous Leishmaniasis [3].

For the diagnosis of mucosal Leishmaniasis, the clinical history and typical cutaneous scars have been considered as important clinical markers to corroborate the diagnosis of LM in patients with non-specific nasal/ oral granulomatous lesions [20].

In the Americas, pre-Colombus pottery, made by the Indians of Peru, has been found, dating from 400 to 900 AD. These show mutilations of lips and noses, characteristics of espundia, today known as muco-cutaneous leishmaniosis. Subsequently, through studies in paleomedicine, mummies with skin lesions and mucosas characteristic of Leishmaniasis were found [9]. Historical findings suggest that American Cutaneous Leishmaniasis (ACL) already affected the peoples of America before contact with Europeans and Africans. It is assumed that it may have originated in the western Amazon region during archeological times by means of human migrations and later ascended to the high jungle and then to the hot inter-Andean lands across the frontiers of Bolivia and of Peru with Brazil [5].

In the Old World (Asia, Africa and Europe) written accounts of the disease date from the first century AD. About two thousand years later, in 1903, the agent of the disease is described for the first time and separately by LEISHMAN and DONOVAN. The disease was visceral Leishmaniasis and its agent, the species now known as Leishmania donovani [4].

The first reference to American Tegumentary Leishmaniasis (ATL) in Brazil is in the document of the Religious Political-Geographical Pastoral 1827, quoted in Tello's book entitled "Antiguidad de la Syfilis en el Peru", where he recounts the journey of Friar Don Hipólito Sanches de Fayas y Quiros de Tabatinga (AM) to Peru, which crossed the regions of the Amazon basin [9]. In 1911, GASPAR VIANNA gave the parasite found by Lindenberg the name Leishmania brasiliensis, because he considered it morphologically different from Leishmania tropica. This characterized, from then on, the etiological agent of the disease being referred to as "Bauru ulcer", "angry angry" or "tapir-nose" [5].

In the 80s, the ATL was noted in 19 Federative Units (i.e. states), its geographical expansion being verified when, in 2003, autochthony was confirmed in all Brazilian states. It is seen to be widespread and, in some areas there is an intense concentration of cases, while in others, there are isolated cases [7]. The disease has been described in almost all American countries, from the extreme South of the United States to the North of Argentina, with the exception of Chile and Uruguay [22].

\section{Epidemiology}

Leishmaniasis is the second most common parasitic disease in the world, with an estimated 600,000 new cases per year [6]. It can also be considered an occupational disease, since it has affected workers in mining areas, geologists, scientific expeditions, military personnel in training [2]. It has been documented in several countries, with an estimated preva- 
lence of 12 million worldwide [3]. More than 20 Leishmania species pathogenic to man have been described.

Until the 1990s, the classification of these species was based primarily on clinical and geographic criteria, taking into account on the one hand, the distinction between Old and New World, and, on the other, the clinical forms of the disease. This type of classification which has been progressively replaced by phylogenetic classification, is seen to be increasingly less tight and more superficial [4].

Leishmaniasis species are widely distributed and have been documented in Africa, Europe, Asia and America. In the Old World, L. tropica, L. major and L. aethiopica, which cause tegumentary Leishmaniasis, are identified as causal agents of the disease. In the Americas, several species of Leishmania are capable of causing tegumentary Leishmaniasis, such as L. braziliensis (LVB), L. amazonensis, L. guianensis, L. pananmensis and L. Mexicana.

The characterization of Leishmania species that was initially made, considering the behavior of the parasite in the vector, today has biochemical and imminological and molecular biology techniques, by isoenzyme analysis, reactivity with monoclonal antibodies and analysis of the DNA of kinetoplast [3]. In the Americas, 11 dermotropic species of Leishmania causing human disease are currently recognized and 8 species desrcibed as being only in animals [8].

It is in Brazil that the largest prevalence in the whole American continent is found, this being estimated as 65,000 new cases per year [6]. The coexistence is observed of a double epidemiological profile, expressed by the maintenance of cases coming from old foci or areas near them, and by the appearance of epidemic outbreaks associated with factors arising from the emergence of economic activities, such as mining, expansion of agricultural frontiers and extractivism, in environmental conditions that are highly favorable to the transmission of the disease [8].

A great number of the houses in recent population settlements are built very close to the edge of the forest and individuals are affected by the radius of action of these vectors that reach houses and are also attracted by several factors such as lighting, the presence of sinantropic animals such as Didelphis marsupialis, domestic animals and man himself [1]. Some species of rodents, marsupials, edentates and wild canids have already been recorded as hosts and possible natural reservoirs. The reservoir-parasite intersection is considered a complex system, insofar as it is multifactorial, unpredictable and dynamic, and forms a biological unit which can be in constant change as a result of the changes in the environment [8].

The disease occurs, more habitually, in the form of epidemic outbreaks. Thus, the degree of exposure of the individuals affected is related directly to agricultural population settlements which were planned or more often arise from occupation processes on the outskirts of a city, most of which are disorganized [1].

Initially, the reservoirs of the mosquito transmitter were in the wild or in rural areas, but the environmental transformations, provoked by the migratory process and by the increasing urbanization have been modifying this profile. The adaptation of the vectors to the new conditions enabled the disease to spread in the domiciliary and peri- domiciliary settings [5]. 
In several regions of the country, such as in the South and Southeast, intense environmental changes occurred due to anthropic action and agricultural and pastoral activities, which led to the near disappearance of cutaneous Leishmaniasis in the late 40s. However, from the 70s and 80s Leishmaniasis has reappeared in these regions, with a significant increase in the number of new cases arising from endemic areas [1]. Transmission classically was due to the bite of an insect, the so-called insect vector. This insect, also called a sandfly, belongs in the Old World to the genus Phlebotomus, and in the New World, to the genus Lutzomyia [4].

The first cases of ATL in America date from 1885 and in Brazil, the first report was in 1909. In the period 1985-1999, there were 388,155 auctothon cases in Brazil of ALT; from 1999 to 2003, 33,872 cases of ATL a year were registered [1]. In the period from 2000 to 2009, an average of 24,684 confirmed cases of Leishmaniasis was registered in Brazil the Information System for Notifiable Diseases (SINAN) [14]. In 2003, the regions with the highest prevalence of LTA were the North (14,200 cases) and the Northeast (8,005 cases) [5].

In Brazil, 23,399 confirmed cases of ATL were notified in 2009, of which 94.1\% were new cases and $4.6 \%$ relapses. With respect to clinical manifestations, $93.7 \%$ of patients had the cutaneous clinical form and $6.2 \%$, clinical mucosa. Of all patients, in 2009 , only $73.5 \%[17,23]$ were cured, 16 patients died due to ATL, and 122 died from other causes, noting that $21.2 \%$ there was no information on the evolution of $21.2 \%$ of the cases [14].

It is estimated that every year there are new cases in Brazil and the growth of this number is due in part to the emergence and growth of AIDS and deforestation areas [2]. It mostly affects young and adult males [16]. The greater number of cases of American Tegumentary Leishmaniasis among men and adults suggests extra-domiciliary transmission in the economically active population, while this occurrence among women, children and people with nonagricultural occupations suggests intra- and or peridomiciliary transmission.

The transmission of ATL in the Amazon presents a clear seasonal variation, it being more intense in the rainy season, when the temperature, solar radiation and evaporation are lower and humidity higher, thus favoring an increase in the density of the phlebotominae, including the species involved in the cycle of the disease [1]. In endemic areas, there may be significant percentages of children with the disease [16].

\section{Etiological agents and vectors}

In the Americas, 11 dermotropic species of Leishmania which cause the disease in humans are currently recognized and 8 species have been described as affecting only animals. However, in Brazil, 7 species, there being 6 of the subgenus Viannia and 1 of the subgenus Leishmania, have been identified. The three main species are: Leishmania ( Leishmania) amazonensis - distributed throughout the primary and secondary forests of the Amazon (Amazonas, Pará, Rondônia, Tocantins and the southwest of Maranhão southwest), particularly in igapó and forest areas of the "swamp-forest" type. Its presence extends to the regions of the Northeast (Bahia), Southeast (Minas Gerais and São Paulo) and Midwest 
(Goiás); Leishmania (Viannia) guyanensi - apparently limited to the north of the Amazon Basin (Amapá, Roraima, Amazonas and Pará) and extending to the Guianas. It is found mainly in terra firme forests in areas that do not flood during the rainy season; Leishmania (Viannia) braziliensis - is widely distributed, from the South of Pará to the Northeast, also reaching the center-south of the country and some areas of the Eastern Amazon East. In the Amazon, the infection is usually found in dry land areas.

As the subgenus Viannia, there are other species of Leishmania that have been recently described: L. (V) lainsoni, L. (V) naiffi with a few human cases in Pará; $L .(V)$ shawi, with human cases found in Pará and Maranhão. More recently, the species L. (V.) lainsoni, L. (V.) naiffi, L. (V.) lindenberg and L. (V.) shawi were identified in states of the North and Northeast regions [8]. In areas of transmission of L. braziliensis concomitantly or after resolution of the cutaneous disease, about $3 \%$ of patients with cutaneous Leishmaniasis will develop the mucosal form of the disease [3].

Mucosal Leishmaniasis (ML) is a form of tegumentary Leishmaniasis associated with $L$. braziliensis, L. panamensis and less frequently with L. amazonensis [11]. The vectors of ATL are insects known as phlebotominae, belonging to the order Diptera, Psychodidae family, sub-family Phlebotominae, genus Lutzomyia, popularly known, depending on the geographical location, as straw-mosquito straw, tatuquira, birigui, among others [8]. Generally not exceeding $0.5 \mathrm{~cm}$ in length, with long and spindly legs and a dense body follicles. A characteristic of theirs is a hopping flight and keeping their wings erect, unlike other dipteral [9]. The vectors are Lutzomya anduzei, Lutzomyia whitmani and Lutzomyia umbratilis, which is the main vector, which usually lands during the day on tree trunks and attacks people in large numbers, when disturbed [16]. It is usually brownish ("straw-mosquito"), only the females being adapted with the respective mouth part to prick the skin of vertebrates and suck blood.

The genus Lutzomyia is responsible for the transmission of Leishmaniasis in the Americas, and there are 350 species catalogued, which are distributed from the South of Canada to the North of Argentina. Of these, at least 200 occur in the Amazon basin. Very little is known of their breeding grounds, with immature forms being found in debris of rock crevices, caves, roots of the soil and of dead and damp leaves, and also in the forks of trees in animal burrows - ie in moist but not wet soil, and in debris rich in decaying organic matter [9].

\section{Clinical state}

Tegumentary Leishmaniasis is more common than the visceral disease and is characterized in its classical form by the presence of a well-bounded ulcer with raised edges. [17] ATL is an initial infection of the skin (its site of preferential location) from which it can undergo propagation or a secondary process which goes on to manifest itself in the mucosae of the upper airways [5]. The incubation period of the disease in humans is, on average, 2 months, it being possible for there to be shorter (two-week) periods and longer periods (of up to 2 years) [16].

The disease breaks out, in general, during the first five years that follow the appearance of the skin lesion, but may do so even a few decades after the primary cutaneous lesion, the scar from 
which can still be seen. However, in some patients the disease appears primarily in the mucosa membrane, without leaving traces on the skin. [2]. The most common manifestation is leishmaniotic ulcer: a single skin ulcer or only in small numbers, with raised edges, framed and the absence of local pain. Other morphological features can also be identified, such as: infiltrated plaque, tubercule, nodule and verrucous vegetating lesion. When the mucosa is injured, it can present an infiltrated erythema, granular or ulcerated aspect. In order of frequency, mucosal lesions are manifested mainly in the nose, hard palate, pharynx and larynx, which they can present themselves with an erythematous-infiltrated, granular, ulcerated or polypoid aspect with a roughly rounded surface [5].

Basically, it is possible to do the staging of the lesions that have occurred in the ATL by taking into consideration the time of onset, extent and spread of the lesion, and grouping them into: 1). Primary infections: which characterizes the primary accident (initial injury) or leishmaniotic cancer, found at the site of the bite, and after the incubation period (two weeks to one year), erythematous papules appear that progress to forming ulcers with serosanguinous crusts. 2) The onset of secondary Leishmaniasis ranges from one to three months after the primary infection, involving the skin, lymph nodes, lymphatic organs and mucosa and by contiguity, the mucous membranes of the nose, lips, eyelids and genitals are affected when the primary or secondary lesions settle near these regions. 3) Tertiary Leishmaniasis requires a longer period to appear, generally, five to ten years after the initial lesion and is characterized by the presence mainly of naso-oro-pharyngeal, laryngeal and ocular lesions, and it is in this period tertiary that the primary infection has already disappeared and the secondary one, in general, is still present [5]. The clinical presentation exhibits polymorphism and the spectrum of severity of the signs and symptoms is also variable, although there is a certain correspondence between the different clinical presentations and the different species of the parasite [7].

Mucosal Leishmaniasis appears under the following clinical forms: 1) Late -this is the most common form. Classically, it is associated with multiple or long-lasting skin lesions, spontaneous cures or insufficient CL treatments; 2) Of undetermined origin - when the ML is clinically isolated, it not being possible to detect any other evidence of prior CL; 3) Concomitant - when the mucosal lesion occurs at a distance, but at the same time as the active skin lesion (not contiguous to the natural orifices); 4) Contiguous - this occurs by direct propagation of the skin lesion, located next to natural orifices, to the mucosa of the aerodigestive tracts. The skin lesion may be in activity or healed at the time of diagnosis; 5) Primary - this occurs, possibly, by the bite of the vector in the mucosa or semimucosa of lips and genitals [7.8].

It is believed that untreated mucosal lesions are progressive, there being few reports of spontaneous cicatrizations of these lesions which, even if treated, may leave permanent sequelae [22]. There are several hypotheses that attempt to explain the predilection of the nasal mucosa: direct contact of the hand with the skin lesions and scratching one's nose afterwards, the epithelium of the anterior part of the nasal cavities offer conditions to the location of Leishmanias and the lower temperature in the anterior area of the nasal septum, due to the presence of a current of inspiratory air. The transition zone between the squamous epithelia and the pseudostratified vibrating, in the anterior part of the nasal septum and the lower turbinate head, is the "locus minoris resistentia" to the Leishmaniasis process. However, the most 
consistent hypothesis says that Leishmania requires lower temperatures for its growth. Thus, since the anterior area of the nasal septum is cooler due to the inspiratory airflow, there would be a predilection for proliferation of the parasites [13]. The association of low temperature with Leishmaniasis may, in part, be explained by the documentation in vitro that macrophages grown at $29^{\circ} \mathrm{C}$ are less able to destroy Leishmania than macrophages cultured at $33^{\circ} \mathrm{C}$ [3].

It occurs more often in males and at age bands usually higher than CL, which is probably due to its character of secondary complication [8]. The involvement of the nasal and/or mucosa is usually more severe and thus may cause sequelae and death [20].

The initial lesion is characterized by a whitish nodulation without ulceration which is usually observed in the cartaliginous septum (Kiesselbach's area), the floor and side wall, specifically on the head of the inferior turbinate. This impairment classified as stage I of the disease, represents a very early stage of inflammation and does not resemble, from the clinical standpoint, any other nasal disease. Subsequently, a fine granular lesion appears, characterized by superficial ulceration, documented at the anterior septum, inferior turbinates and floor of the nasal cavity (Stage II) [17]. At first, there is hyperemia and edema of the mucosa of the anterior septum, with the establishment of nodulations [3].

In Stage III, or the stage of deep ulceration, tissue reaction is more intense, with aberrant granulation tissue and infiltration of the mucosa, thus widening the nasal septum. There is sometimes edematous infiltration of the nasal pyramid itself. In this phase, hematic crusts can be observed on the septum, the inferior turbinates and the floor of the nasal cavities. These lesions are characterized by excessive fragility, as they bleed very easily when the mucosa is touched.

Clinically, the patient may complain of soreness at the level of the nasal pyramid, sanguinolent rhinorrhea and emission of hematic crusts. Nasal obstruction is a frequent symptom in this phase of the disease. From the external point of view, due to the inflammatory process that involves the cartilages and subcutaneous cell tissue and the very skin of the base of the nasal pyramid, the nose takes on the aspect known as tapir-nose.

Stage IV of the disease is characterized by the cartilaginous involvement of the anterior septum with necrosis and, sometimes, impairment of the columella. It is at this stage that perforation of the cartilaginous septum is established, also with marked infiltration of the posterior septum. In more advanced forms (Stage V), total destruction of the columella may occur and may drop, thus transforming the nasal cavity into a similar cloaca and sometimes there is perforation of the dorsum of the nasal pyramid [17]. For some researchers, the specific destruction of the nasal cartilage could also indicate an autoimmune reaction that would explain why some patients undergo severe tissue destruction and others only present mucosal involvement years later [21]. In some cases there may be total destruction of the anterior septum, only the entire columella remaining, with the nose being sealed. Extensive crusts of a hemorrhagic aspect or even resulting from the drying of mucous secretion caused by enlargement of the internal nasal structure can be observed as a consequence of the tissue injury, represented by the destruction of the cartilaginous septum and inferior turbinates. On this occasion, there is elimination of sanguinolent discharge and 
the presence of crusts is accentuated [17]. The patient's death usually occurs because of aspiration or respiratory failure [5].

The earliest signs and symptoms of mucosal Leishmaniasis are nasal obstruction, epistaxis and the establishment of granulomas in the anterior nasal septum. As the disease evolves, patients begin to present a leishmaniotic facies known as "tapir nose" due to edematous infiltration of the lining and supporting structures of the nose [3]. Lesions reach the cartilaginous nasal septum and may extend to the lateral wall and floor, the region of the palate, uvula, and less frequently, involvement of the pharynx, larynx, vocal cords and upper lip occur with varying degrees of infiltration, granulation and ulceration [17]. The infiltration of the soft palate reaches the proportions of a real tumor. The whole palate is changed: the uvula is reduced to a shapeless mass, with an irregular, vegetating surface. In the palatal vault, lobed prominences are formed, separating themselves by sinuous furrows and ulcerated erosions.

More rarely it can involve the gum and dental interstices, where voluminous and prominent granulations develop, and reach the upper lip. The tongue is usually spared [5]. The manifestations of the mucosal diseases include involvement of pillars and uvula with an increase in volume, hyperemia, roughness and superficial ulcers [12]. The pharynx is the second site of involvement when mucosal lesions caused by L. braziliensis set in. As in the nose, the lesion initially observed at the level of the mucosa of the pharynx takes on a lumpy aspect; however, here, there is a much more intense edematous infiltration, especially of the uvula and secondarily of the tonsillar pillars, extending also to the mucosa of the posterior pharyngeal wall.

The appearance can be observed at this stage of granulation tissue that is a little redundant intermingled with the lumpy aspect of the mucosa. The next stage is characterized by the appearance of abundant granulation tissue, which causes important tissue destruction, also involving the lymphoid tissue of Waldeyer's lymphatic ring at the level of tonsils. Areas with a tenuous fibrin layer mix in with the granulation tissue of a vegetating aspect.

Because of the intense tissue aggression, in the specific post-treatment healing process, the presence of abundant fibrous tissue, with the formation of true whitish cords can be documented. These completely deform the configuration of the anatomical structures of the velum of the palate and the posterior wall of the pharynx, leading to full stenosis in the communication of the oropharynx with the nasopharynx [3]. In the mouth, the hard palate is often involved, with dissemination of the process to the soft palate, uvula and pharynx. The proliferating infiltrative process can cause fusion of the uvula, pillars, lateral cords and posterior wall, causing obliteration of the nasopharynx. Deformity and narrowing of the lumen of the oropharynx may occur due to fibrosis of the tonsils [5].

Laryngeal mucosa is the $3^{\text {rd }}$ site of election when mucosal Leishmaniasis sets in. As in the pharynx, the mucosal lesions present the same characteristics of finely granular tissue. There may be in situations of greater inflammation, the presence of granulation tissue with a vegetating aspect, covered at times with a tenuous fibrin layer, involving the mucosa that covers the cartilage of the epiglottis, extending to the mucosa of the structures of the laryngeal vestibule and vocal folds. At this stage, dysphonia characterized by a muffled voice is always present, which draws attention to the impairment of the organ [3]. Pharyngolaryngeal 
involvement can be intense, to the point of causing dysphagia, dyspnea, dysphonia, odynophagia, and coughing [15]. The hypopharynx, larynx, epiglottis, arytenoid cartilages and the posterior commissure of the vocal cords are covered by a lesion with a vegetating aspect, which sometimes come to join up. These granulations often regress and eventually disappear, making the surface affected by a smooth and slightly whitish coloration.

There is generalized laryngeal inflammation particularly in the piriform sinuses. The vocal folds appear to be moving well, but phonation is weak and the muscular control of tension can be harmed by granulomatous formation and subsequent fibrosis. Even after successful treatment, the voice rarely returns to normal and the lumen of the larynx may be reduced [5]. Painful dysphagia in degrees of greater or lesser intensity prevents the normal feeding of the individual, with consequent impairment of general condition and, in very advanced cases, becoming cachexia. In post-treatment healing, the deformities that these cartilages present are very evident, such as fibrous tissue which is also whitish, thereby completely modifying the anatomy of the organ, except for a residual permanent dysphonia [3].

Complications include pneumonia due to aspiration, bacterial infections, secondary myiasis, cachexia due to difficulties in swallowing, laryngeal edema and asphyxiation, which may be lead on to the patient's death mainly due to respiratory failure and sepsis [5]. The presentation of the clinical form with lesions exclusive of mucosa of the larynx and trachea is relatively uncommon [2]. The ear is not usually affected in mucosal Leishmaniasis. However, the involvement of the mucosa of the nasopharynx leads to impairment of the pharyngeal orifice of the Eustachian tube, situated on its sidewall. A process of otitis media with effusion (secretory otitis media chronic) can be established in these cases.

The sensation of blocked ear, tinnitus and hearing loss are complaints in these cases [3]. Morbidity of the skin and ear cartilage occurs because it is a place of lower temperature, apt for the growth of Leishmania, besides being an area exposed to the inoculation of the vectors. The external ear commonly presents an increase in volume, ulcers with raised edges, sometimes covered with crusts, and may appear as an infiltrated plate, tubercule, vegetating warty nodule and lesion, on course in the end to mutilating the ear [5].

Mucosal Leishmaniasis can compromise the labial mucosa and gingival margin. This is a rarer manifestation of the disease [3]. The lesion in some individuals heals early, sometimes without seeking medical attention. Others remain for months with the lesion in activity and the healing process is slow. This phenomenon can be explained by the rapid or late establishment of a specific immune response which is effective in eliminating the parasite.

The cure of Leishmaniasis is not sterile. It has been possible to isolate viable parasites of ALT scars in individuals who have been cured for several years, a fact confirmed in experimental studies using an animal model. This phenomenon could thus explain the appearance of late relapses as well as the onset of the disease in immunocompromised patients, such as AIDS (Acquired Immunodeficiency Syndrome) [7]. 


\section{Diagnosis}

It is very hard to detect Leishmaniasis in the initial stage [15]. The long interval between the onset of symptoms and etiological diagnosis of the mucosal form of ATL may reflect the limitation of the training of most physicians in the proper approach to mucosal Leishmaniasis [2]. A laryngoscopy exam usually demonstrates an extensive inflammatory component, with erythema and edemas evident. The granulomatous aspect associated with the presence of ulcers is common, and may present purulent exudate. In the advanced disease, tissue destruction can be striking. As a protocol of etiological investigation on suspicion of granulomatous bodies that are difficult to access such as the larynx, laboratory tests and imaging should be requested, and should a diagnostic uncertainty be maintained, a biopsy of the lesions is recommended for histological study. If the appearance of the lesion suggests malignant neoplasm, research using noninvasive and invasive tests should occur simultaneously so as not to delay diagnosis [15].

The ENT examination associated with the Montenegro test remains the most important element for diagnosis, although it is usually of a presumptive character [20]. The encounter of Leishmania is the gold standard for the diagnosis of ATL [21]. The diagnosis can be confirmed by various tests: 1) Direct investigation of the parasite, which can be done by scraping the ulcerated surface or by compression of the slide on the wounded area of the lesion. The material is stained with Giemsa or Wright [2]. The direct parasitological examination is the procedure of first choice because it is faster, less expensive and easy to perform [16]. It gives good results in initial lesions, without associated bacterial infection [2], 2) Montenegro intradermoreaction: This translates the response of cell delayed hypersensitivity [16]. It consists of intradermal injection of $0.1 \mathrm{ml}$ of antigen prepared from Leishmania promastigotes, with a reading after 48 hours. The test is considered positive that produces an induration of $5 \mathrm{~mm}$ or more. However, the positivity of the test indicates that the person has already been sensitized but is not necessarily a carrier of the disease [2]; 3) Histopathological examination of the tegumentary lesion [2]. The Biopsy can be performed with a "punch" of $4 \mathrm{~mm}$ in diameter, or a wedge, with the use of a scalpel. In ulcerated lesions, the whole edge of the whole lesion should be preferred, This, in general, shows a tumified and hyperemic aspect [16]; 4) Serology (indirect immunofluorescence or ELISA); they have good sensitivity but can give a reaction crossed with Chagas disease and visceral Leishmaniasis, this being the cause of false-positive results, thus reducing its specificity [2].

The most commonly used techniques for antibodies are: indirect immunofluorescence (IIF), counterimmunoelectrophoresis (CIE ), ELISA and Western blot. The Western blot technique has a superior sensitivity to the other serologic techniques [70.6\%), a sensibility of $70.3 \%$ and a precision of $72.7 \%$. In the immunocompetents, the specificity and sensitivity are $100 \%[4] ; 5$ ) Immunohistochemical techniques (immunostaining with anti Leishmania antibodies); they permit evidence of the parasite in histological sections; 6) Method of culture: culture takes place in Novy-MacNeal-Nicolle medium from the biopsy or aspirate [4]. They are not practical for diagnosis, especially of Leishmania brasiliensis, since it does not grow easily in culture media; in addition, bacterial or fungal contamination often complicate this procedure [2]. Research 
can be done into Leishmania in other affected organs such as the spleen, liver and lymph node, and whenever there is a suspected diagnosis, into the pleural fluid, bronchoalveolar lavage, intestinal biopsy, skin, etc. Hepatic and spleen biopsies are used as a last resort due to the increased risk of potentially serious complications such as hemorrhaging [4].

Cases are confirmed according to the following criteria: 1) Residence, arrival in or moving away from the area with confirmation of transmission and presence of the parasite in direct and/or indirect parasitological exams; 2) Residence, arrival in or moving away from the area with confirmation of transmission and intradermoreaction of Montenegro (MRI) positive, 3) Residence, arrival in or moving away from the area with confirmation of transmission with other methods of positive diagnosis [19].

\section{Differential diagnosis}

The finding of symptoms and head and neck moles in patients with Leishmaniasis, paracoccidioidomycosis and leprosy underscore the need that they all undergo an ENT evaluation. In addition to these diseases, it would be an appropriate conduct to perform complete ENT examination in all patients with some form of granulomatous disease [11]. The differential diagnosis of laryngeal ATL is made with granulomatous lesions such as tuberculosis and paracoccidioidomycosis which have a predilection for the posterior portion of the larynx [2]. The diagnosis of paracoccidioidomycosis is characterized by erosion or exulceration in the oral mucosa, with a granulous base and the presence of stippled hemorrhage (Aguiar Puo's moriform stomatitis), regional lymph node and lung involvement [8]. Syphilitic laryngitis, which fortunately nowadays is rare and appears in the tertiary stage of syphilis may present diffuse infiltrate, which subsequently undergoes ulceration [2]. Tertiary syphilis can be confirmed by histopathological exam, and shows vascular lesions and plasma cell wealth, and VDRL may be positive [8].

Neoplastic lesions of the larynx are more localized and almost always there is a report of smoking and alcoholism in the medical history [2]. Epidermoid and basal cell carcinomas usually present themselves as hardened to palpation, and are confirmed by histopathological examination [8]. Histoplasmosis in its chronic disseminated form involves mucosa in $90 \%$, and the upper airways are affected; the larynx is very adversely affected with infiltration and edema of the laryngeal vestibule, pink nodules on an infiltrated base and granulomatous ulcerations of a granulomatous depth partially covered by yellowish-white secretions that may lead to obstructive dyspnea, requiring a tracheostomy. Generally, in this form of presentation of histoplasmosis there will be alterations in the chest X-ray associated with pulmonary symptoms, which differentiates it from Leishmaniasis. Coccidioidomycosis of the larynx is very rare in our environment. Tracheobronchial amyloidosis with involvement of the larynx is very rare; however, it may present itself with pseudotumoral, bleeding, warty lesions, with a visual appearance similar to the case presented, which can lead to obstruction of the airways [2]. The differential diagnosis is made with rhinophyma, rhinosporidiosis, entomophthoromycosis, traumatic septal perforation or because of drug use, allergic rhinitis, sinusitis, sarcoidosis, Wegner's granulomatosis and other rarer diseases. 
As for lymphomas, histopathological and immunohistochemical exams will help to conclude the diagnosis. In the case of rhinophyma, there is usually a history of rosacea (acne-like lesions and telangiectasias, of long evolution). In the differential diagnosis with rhinosporidiosis what are important are: the origin (Piauí, Maranhão), the history of possible exposure to the fungus in stagnant water and dams, the presence of polyps in the nasal and ocular mucous membranes, and upper respiratory tracts. Histopathological examination shows the microorganism (sporangia of 6 to $300 \mu \mathrm{m}$ ). Lesions of entomophthoromycosis present a hardened or woody consistency to palpation and histopathological and mycological exams demonstrate the presence of hyphae and isolation of the fungus in cultur medium. In the differential diagnosis with leprosy, skin sensibility tests, testing of skin bacilli in the lymph of the pinna or lesions and histopathological examination will help confirm the diagnosis. The clinical history is essential when seeking information on personal or family atopy (allergic rhinitis, bronchitis, migraine), on traumatic perforation and use of drugs. Wegner's granulomatosis and sarcoidosis are rarer diseases, and sometimes difficult to confirm. Diagnosis may be aided by observation of the involvement of other organs such as the lungs and kidneys, it being stressed that the histopathology will contribute to the diagnosis [8].

\section{Co-infection Leishmania and HIV}

ATL can modify the progression of the disease due to HIV and immunosuppression caused by this virus facilitating progression of ATL [8]. Acquired immunodeficiency syndrome is caused by a retrovirus of the Lentiviridae family, HIV-1 and HIV-2. Those infected with the human immunodeficiency virus (HIV) progress to severe dysfunction of the immune system, as the CD4 + T lymphocytes, one of the major target cells of the virus, are being destroyed [19]. On destroying the immune system, the so-called "opportunistic infections" are manifested as this unfolds in which infections are included, namely infections by protozoa [4]. The assessment of the set of clinical manifestations of ATL in patients with HIV indicates that there is no definition of a clinical profile that may unarguably be associated with co-infection [8]. The exponential increase in the number of cases of coinfection of Leishmania/ HIV, especially in the late 1990s, has undergone modifications [4]. Unusual findings can be observed in co-infected patients, suchas, for example, finding Leishmania spp in intact skin, and overlying a Kaposi's sarcoma lesion, or Herpes simplex and Herpes zoster lesions. There may also be involvement of the gastrointestinal tract and the respiratory tract for the coinfection of ATL/AIDS [8]. The population of drug users who inject endovenously is the main group at risk of the co-infection of Leishmania/HIV in Southeast Europe, and form $72 \%$ of co-infected patients [4].

\section{Prevention}

Several thorough trials have been carried out on the production of anti- Leishmania vaccines:

1) "Leishmanization" has been used empirically since the distant past by people living in 
endemic areas. This consists of scarification covered with virulent promastigotes of virulent to avoid the appearance of disfiguring lesions in exposed areas. 2) The association of BCG with dead Leishmania promastigotes. 3) Vaccines produced by molecular technology from DNA, probably the future of prophylaxis in infectious diseases in immunosuppressed patients [4].

\section{Treatment}

ENT activity is of primary importance [5]. The drug of first choice is the pentavalent antimonial one. With a view to standardizing the therapeutic regimen, WHO recommends that the dose of this antimonial be calculated in $\mathrm{mg} / \mathrm{SbV} / \mathrm{Kg}$ day./ (SbV meaning a pentavalent antimonial).

There are two types of pentavalent antimonial that can be used, N-methylglucamine antimonate and sodium stibogluconate $[10,16]$. In all forms of mucosal involvement, the recommended dose is $20 \mathrm{mg} \mathrm{Sb}+5 / \mathrm{kg} /$ day, for thirty consecutive days, preferably in a hospital environment. If healing is not complete within three months (twelve weeks) after treatment ends, the scheme should be repeated only once. Should there be no response, use one of the second choice drugs [16].

If there is no satisfactory response to the treatment with pentavalent antimony, the second choice drugs are amphotericin B and pentamidine isethionate. The injections must be made parenterally, intramuscularly (IM) or intravenously (IV), with rest after application. The IM may have the drawback of local pain. It is suggested, therefore, locations be alternated, the gluteal region being preferred.

In cases of malnourished patients with low muscle mass, and those with thrombocytopenia, the intravenous route should be given preference. This is the best route because it allows the application of large volumes without the inconvenience of local pain. The application should be slow (a minimum of 5 minutes), with a fine needle (gauge $25 \times 7$ or $25 \times 8$ ) and without needing to be diluted. To make it possible for rest after administration, it is generally advisable to apply the medication at the end of the day. It is worth noting that there is no difference between the IV and IM routes, with respect to the efficacy and safety of the drug [8].

The use of topical products such as paromomycin and imiquimod, associated or not with parenteral medication, have also presented preliminary satisfactory results, with cure rates ranging between $74 \%$ and $85 \%$ for the former drug, and $90 \%$ for the latter [9]. The imidazoquinoline, approved for the treatment of genital warts, [17] stimulates the Th1 response by increasing the production of TNF- $\alpha$, IFN- $\gamma$ and IL-12. In vitro, presents anti Leishmanial activity because it also stimulates the production of nitric oxide by macrophages, thus decreasing the number of parasites. Paromomycin is an antibiotic that inhibits the mitochondrial activity of Leishmania. rhGM-CSF is a glycoprotein that induces the growth of colonies of granulocytes and/or macrophages, by stimulating their phagocytic and metabolic functions. For this reason, it plays an important role in the immune response against intracellular pathogens. It has been used experimentally in the treatment of some inflammatory diseases as it has an inhibitory effect on TNF- $\alpha$ [18]. 
Local treatment of small lesions may not be necessary. Larger lesions may be treated with surgical excision, curettage or cryotherapy [13]. Secondary infection may occur in $54.2 \%$ of patients and the germ most frequently found is Staphylococcus aureus [21], which is why local care should be prescribed such as local cleansing with soap and water and if possible compresses with $\mathrm{KMnO}$ (potassium permanganate in a dilution of $1 / 5000 \mathrm{ml}$ ) $[8,16]$.

The cure criterion is defined by the regression of all signs and confirmed by ENT examination, up to 6 months after completion of the treatment regimen. In the absence of the specialist, the clinician must be trained to perform, at least, anterior rhinoscopy and oroscopy. Where there is no clinician, the patient should be referred to the service that evaluates healing. The patient should return monthly for a consultation for 3 consecutive months after the end of the treatment regimen so that the clinical cure can be evaluated.

Once cured, the patient should be monitored every 2 months, until 12 months after completing treatment [8]. The control of ATL should be tackled in a comprehensive way, in five respects: epidemiological surveillance, measures of performance in the transmission chain, educational measures, administrative measures and vaccine [9]. To reduce the lethality of these diseases, what is above all necessary is early diagnosis and the timely treatment of cases [14].

The current challenges for ATL are: a) to increase investments in seeking drugs with better efficacy, safety, low cost, ease of administration and sustainability; b) to continue to be vigilant about the adverse effects of medication; c) to expand the health network for early diagnosis and adequate treatment of cases; d) to investigate and evaluate deaths; e) to implement surveillance actions in territorial units; f) to expand the activities of epidemiological surveillance [19].

\section{Acknowledgements}

Our thanks to the Teacher Roddy Kay for his dedication in translation of this chapter.

\section{Author details}

Luiz Alberto Alves Mota ${ }^{1,2^{*}}$ and Roberta Correia Ribeiro Ferreira de Miranda ${ }^{3}$

*Address all correspondence to: luizmota10@gmail.com

1 Faculty of Medical Sciences, Universidade de Pernambuco, Brasil

2 ENT service, Hospital Universitário Oswaldo Cruz, Brasil

3 Faculty of Medical Sciences, Universidade de Pernambuco, Brasil 


\section{References}

[1] Guerra JAO, Barbosa MGV, Loureiro ACSP, Coelho CP, Rosa GG, Coelho LIACR. Leishmaniose tegumentar americana em crianças: aspectos epidemiológicos de casos atendidos em Manaus, Amazonas, Brasil. Cad. Saúde Pública, Rio de Janeiro. 2007, 23(9):2215-2223.

[2] Melo SMD, Todt Neto JC, Andrade LCF. Pseudo-hemoptise por leishmaniose. Jornal de Pneumologia. 1999, 25(6):347- 350.

[3] Lessa MM, Lessa HA, Castro TWN, Oliveira A, Scherifer A, Machado P, Carvalho EM. Leishmaniose mucosa: aspectos clínicos e epidemiológicos. Rev Bras Otorrinolaringol. 2007, 73(6):843-847.

[4] Catorze MGB. Leishmaniose e SIDA. Med. Cutan. Iber. Lat. Amer. 2005, 6:237-250.

[5] Neto FXP, Rodrigues AC, Silva LL, Palheta ACP, Rodrigues LG, Silva FA. Manifestações Otorrinolaringológicas Relacionadas à Leishmaniose Tegumentar Americana: Revisão de Literatura. Arq Int Otorrinolaringol./Intl Arch Otorhinolaryngol. 2008, 12(4):531-537.

[6] Silva L, Costa HO, Duprat AC, Bairão F, Della Nina M. Granulomatose laríngea. Avaliação e métodos diagnósticos e terapêuticos em 24 casos. ACTA ORL/ Técnicas em Otorrinolaringologia. 2007, 25(1):16-23.

[7] Ministério da Saúde do Brasil. Manual de Vigilância da Leishmaniose Tegumentar Americana. 2ª ed. Brasília; 2007, 1-30.

[8] Ministério da Saúde. Secretaria de Vigilância em Saúde. Leishmaniose Tegumentar Americana. Guia de Vigilância Epidemiológica; Caderno 11.

[9] Basano SA, Camargo LMA. Leishmaniose tegumentar americana: histórico, epidemiologia e perspectivas de controle. Rev Bras Epidemiol. 2004, 7(3):328-337.

[10] Secretaria Municipal de Saúde. Recomendações para o Manejo Clínico da Leishmaniose Tegumentar e Visceral. Belo Horizonte; 2007.

[11] Fornazieri MA, Yamaguti HY, Moreira JH, Takemoto LE, Navarro PL, Heshiki RE. Manifestações Otorrinolaringológicas Mais Comuns das Doenças Granulomatosas. Arq Int Otorrinolaringol./Intl Arch Otorhinolaryngol. 2008,12(3):362-365.

[12] Focaccia R, Veronesi R. Tratado de Infectologia. 3ª edição v.2 Atheneu, São Paulo, 1997.

[13] Gomes ACA, Dias EOS, Pita Neto IC, Bezerra TP. Leishmaniose muco-cutânea: relato de caso clínico Rev.Cirurgia e Traumatologia Buco-Maxilo-Facial. 2004, 4(4):223-228.

[14] Tratamento da Leishmaniose Visceral e Leishmaniose Tegumentar Americana no Brasil Epidemiol. Serv. Saúde, Brasília, 20(1):107-110, jan - mar 2011. 
[15] Leishmaniose Laríngea. Arquivos Internacionais de Otorrinolaringologia. São Paulo Brasil, v.16, n.4, p. 523-526, out - dez 2012.

[16] Ministério da Saúde. Gerência Técnica de Doenças Transmitidas por Vetores e Antropozoonoses. Coordenação de Vigilância Epidemiológica Centro Nacional de Epidemiologia. Manual de Controle da Leishmaniose Tegumentar Americana. Fundação Nacional de Saúde Brasília, 2000.

[17] Luiz Henrique Guimarães, et al. Aspectos clínicos da Leishmaniose tegumentar. Gaz. méd. Bahia 2005;75:1(Jan-Jun):66-74.

[18] Almeida OLS, Santos JB. Avanços no tratamento da leishmaniose tegumentar do novo mundo nos últimos dez anos: uma revisão sistemática da literatura. An Bras Dermatol. 2011;86(3):497-506.

[19] Penna GO, Domingues CMAS, Siqueira JB Jr, Elkhoury ANSM, Cechinel MP, Grossi MAF, et al. Doenças dermatológicas de notificação compulsória no Brasil. An Bras Dermatol. 2011;86(5):865-77.

[20] Diniz JLCP, Costa MOR, Gonçalves DU. Mucocutaneous Leishmaniasis: clinical markers in presumptive diagnosis. Brazilian Journal of Otorhinolaryngology 77 (3) Maio/Junho 2011;77(3):380-4.

[21] Silva MS, Sousa RT, Silva EB, Guerra JAO, Gomes NM, Santana RF, Mubarac RS. Primary lesion of Mucocutaneous Leishmaniasis simulating external otitis.

[22] Nunes CS, Yoshizawa JK, Oliveira RZ, Lima AP, Oliveira LZ, Lima MVN Leishmaniose mucosa: considerações epidemiológicas e de tratamento. Rev bras med fam comunidade. Florianópolis, 2011 Jan-Mar; 6(18): 52-56. 